\title{
Application And Research Of UML On Modeling And Simulation In Command Information System
}

\author{
Jiang Miao, Liu Bo \\ Electronic equipment test center \\ LEETC \\ Luoyang China \\ 469480414@qq.com, masterofchikang@sina.com
}

Keywords : UML; Command information system; intelligence Processing; Simulation model

\begin{abstract}
Having analyzed the function of the intelligence processing module in command information system, this paper not only designed both static structure model and dynamic characteristic model for command information system with the method of $\mathrm{OO}$, but also described the models using use case diagram, class diagram, activity diagram and sequence diagram, then built up intelligence processing model which could be better reused for distributed simulation of command information system.
\end{abstract}

\section{Introduction}

Command information system which consists of command, control, communication, computer, intelligence and synthetically using computer and network as the core of information technology, is designed for commanders to command and control in missions of every range. It supplies timely, integrated and correct information for commanders and combatants, so that they can make correct decisions quickly.

As a language in software modeling, UML has the powerful ability in OO software engineering; it supplies different types of frame-diagrams through standard designing process of simulation and modeling. The frame-diagrams describe the whole process which starts from model requirement analysis and ends at realization of model software, also describe requirements and function characters of simulated model through static structure and dynamic behavior in order to satisfy the needs of communication among users ${ }^{\mathbf{I} 3,4 \mathbf{I}}$, analysts, developers, testers, managers and other correlative partners, and finally make it possible to establish a visualized, standardization, structural, documental simulated models.

There are three methods to realize visualized modeling by UML at present: manual technic-examination, formalization method, regularization method. This thesis introduces how to analyze command information system and design its model with UML, how to describe requirement, structure, function as well as corresponding behavior of the models inch by inch, so that developers could comprehend system models which would be designed by themselves perfectly. This paper takes intelligence processing function module, one of command information system's function modules, as an example, to show the application of UML.

\section{Intelligence processing function of command information system}

Intelligence processing function of command information system is used to make synthesized disposals such as storing multi-variable of information from all kinds of detecting instruments or artificial obtainment, summary, classification, deleting repetition, and wiping off contradictions, etc. It mainly offers intelligence production for informative services on the base of requirement from missions.
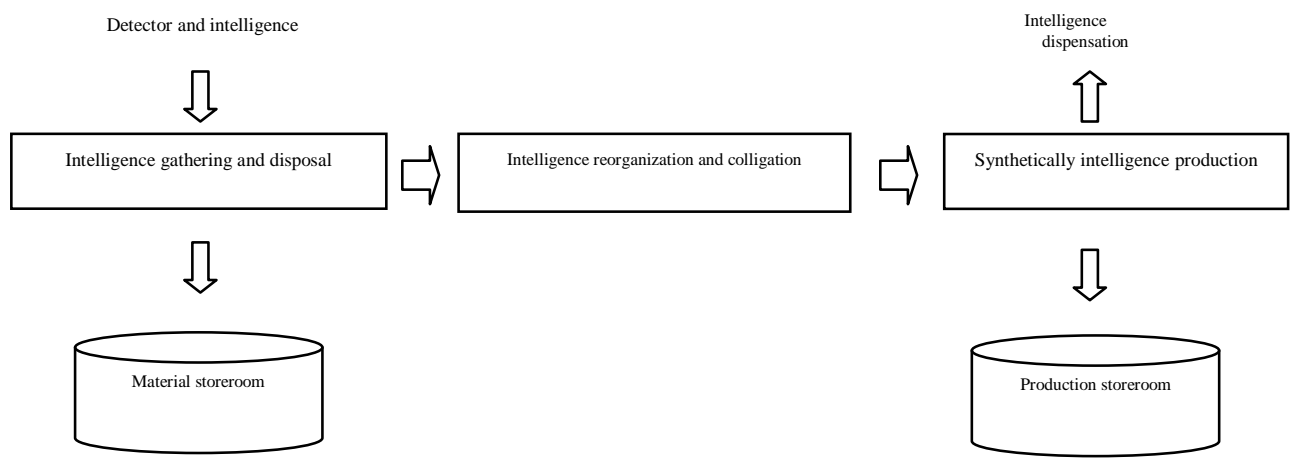

Fig. 1 Sketch map of Intelligence processing function

Fig. 1 demonstrates the model running process based on intelligence processing function as: receiving aerial intelligence, detecting and calculating aerial intelligence in extrapolation to judge whether the target is detected or not, producing radar intelligence, displaying radar intelligence upon the interface, exporting radar intelligence in stated 
format.

state, extrapolation of target state, simulation of detective function, data processing, export and display of intelligence,

Intelligence processing model mainly consists of seven data record, etc.

function modules as shown in Fig. 2: control of simulation

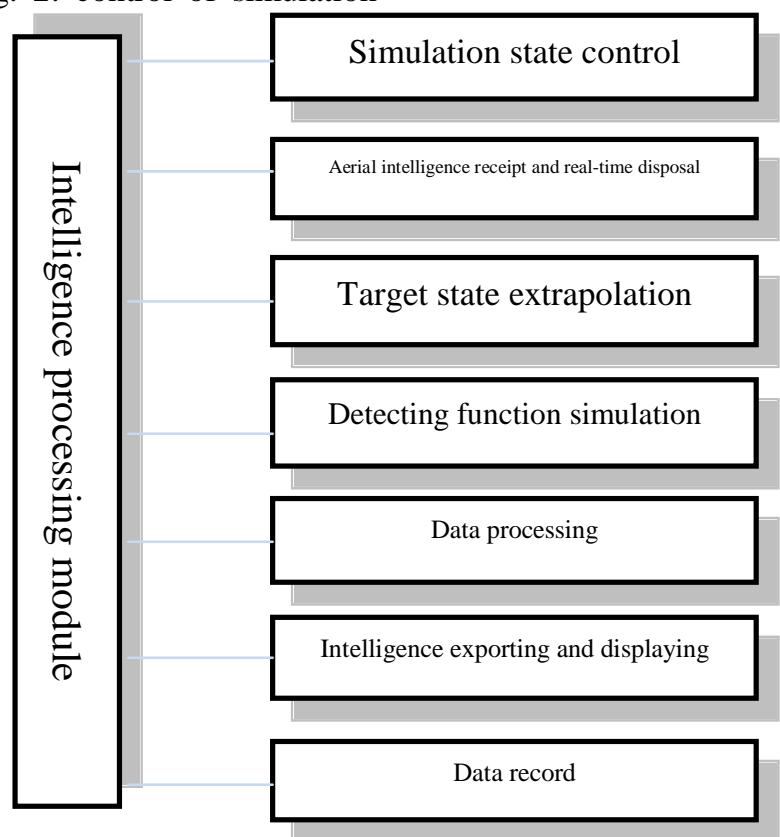

Fig 2 Composing diagram of intelligence processing function module

Diagrams of use case of UML can describe intelligence processing function modules and the interrelation among its composing intuitively and clearly. Intelligence processing use case in Fig.3 describes intelligence processing function and the interrelation between it and its subordinate units.

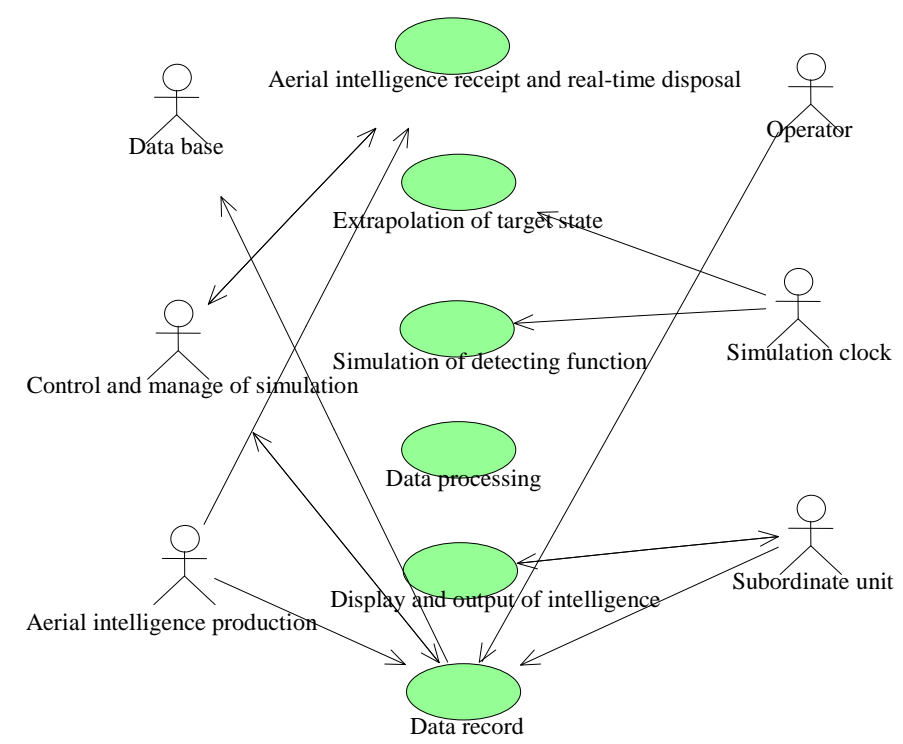

Fig 3 Intelligence processing use case

\section{Description of static structure}

Adopting hierarchical structure to organize models can realize reusing and mutual operation of simulation model. UML adopts class diagram to describe hierarchical structure. Class diagram which considers objects from the static point of view, is an expression of static view, and makes models for entities as well as internal entities which are correlative to the realization.

Taking the realization of intelligence processing use case for example; its class diagram is depicted as Fig 4. 'Paramcompute' is for parameter calculation, and to calculate the integrated aerial parameters (include intimidation degree). 'AirInfoReceiver' is for intelligence reception class, and it charges picking-up intelligence from buffer. 'AirInfoChecker' is for checking aerial-intelligence dot, and it charges checking the correctness of aerial-intelligence dot parameters. 'Trace' is for track, and it describes external track which is incepted from aerial intelligence. 'FuseDeal' is for colligating batches, and it is used to colligate batches of sub-intelligence. These classes describe intelligence processing function of command information model more detailed, to realize the function of intelligence processing use case. 


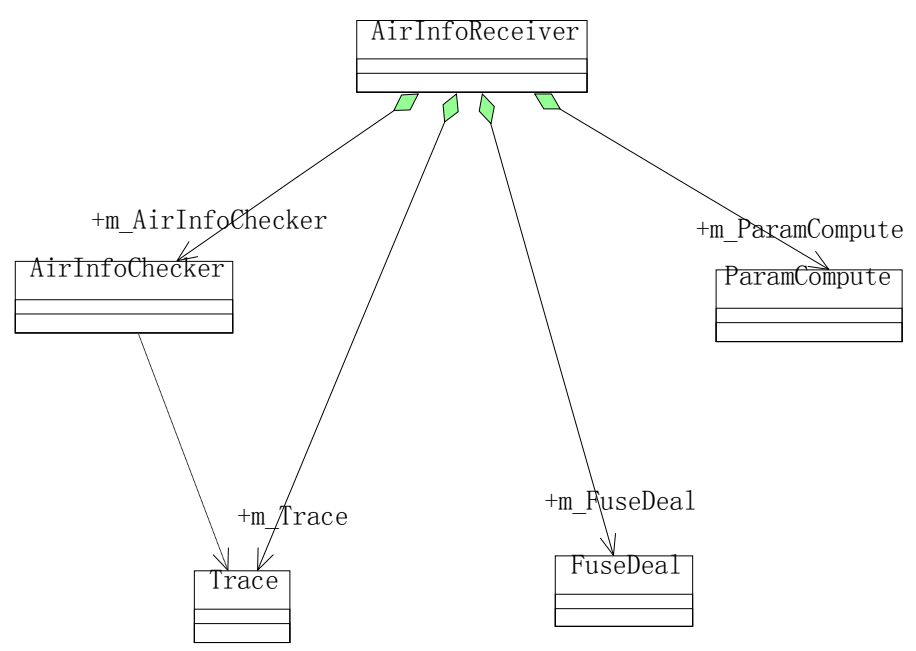

Fig 4 Class-diagram of intelligence processing

\section{Description of dynamic behavior}

UML adopts state-diagram, activity diagram, and sequence-diagram and collaboration diagram to make models for system dynamic characteristic. Static-diagram is equivalent to activity diagram, sequence-diagram and collaboration diagram in semasiology. Sequence-diagram pays attention to time-sequence of messages; each object communicates through messages in $\mathrm{OO}$ techniques, and sequence-diagram describes time-sequence of communication among each object which is the real operated data in program. Collaboration diagram focus on the structure of objects which receives or sends messages and the distributing of disposal processing; it shows the relation between mutual objects. State-diagram pays attention to every change mode of modeling objects' state which is driven by incident. Activity diagram pays attention to control flow from activity to activity; it shows the steps or logic flow when carrying out some operations. This paper mainly takes activity diagram and sequence-diagram for considerations, to explain the description method of simulation dynamic characteristic of the intelligence processing model.

\section{A. Active-diagram of intelligence processing model}

Intelligence processing model receives aerial intelligence, detects and calculates aerial intelligence in extrapolation to judge whether the target is detected or not, produce radar intelligence, display radar intelligence upon the interface, export radar intelligence in stated format. Active-diagram of UML describes this procedure in Fig. 5.

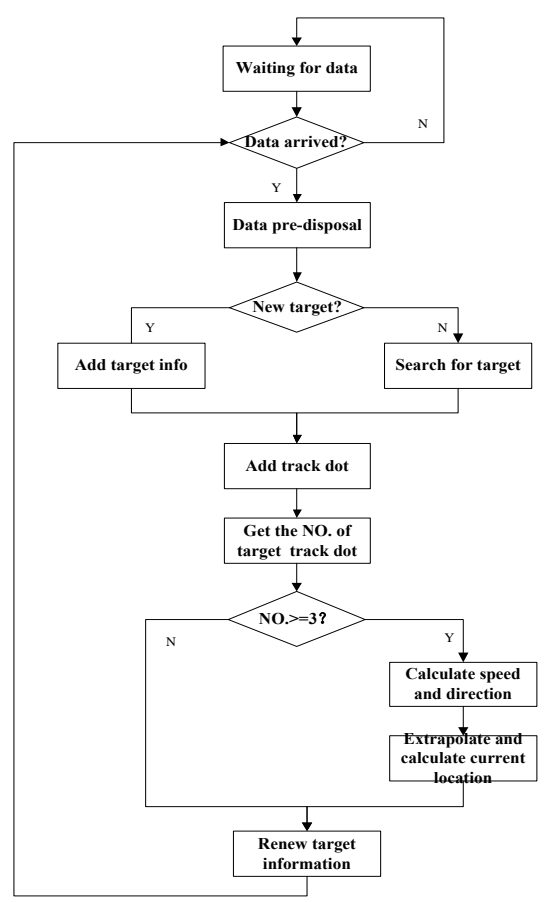

Fig 5 Active-diagram of intelligence processing model

\section{B. Active-diagram of intelligence processing model} receiving and real-time disposal aerial intelligence

Connecting and real-time processing aerial intelligence is the most important gist to form and export timely, effective radar intelligence, and it mainly contains: the model of advanced watchful radar aerial targets; to judge whether the target is detected or not; if the target is detected, the program will calculate and read data; then do some correlative disposal; and display and output intelligence. The state of operation implement is described as following Fig.6.

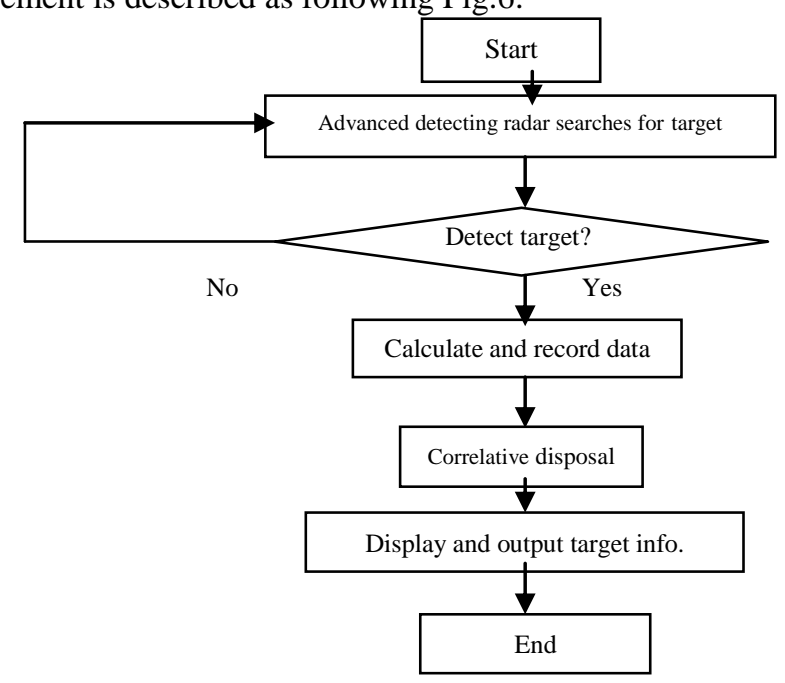

Fig 6 Active-diagram of receiving and real-time processing aerial intelligence

\section{Sequence-diagram of intelligence processing}

Sequence-diagram mainly describes the relationship of transmission and transfer of messages which between 
simulation objects. Fig.7 takes sequence-diagram of intelligence processing for example, and describes the sequence of buffer managing, radar thread, information managing and displaying upon the interface. The topmost rectangular round describes the objects and roles which participate in the realization of this function. The arrow between two objects shows their communication.

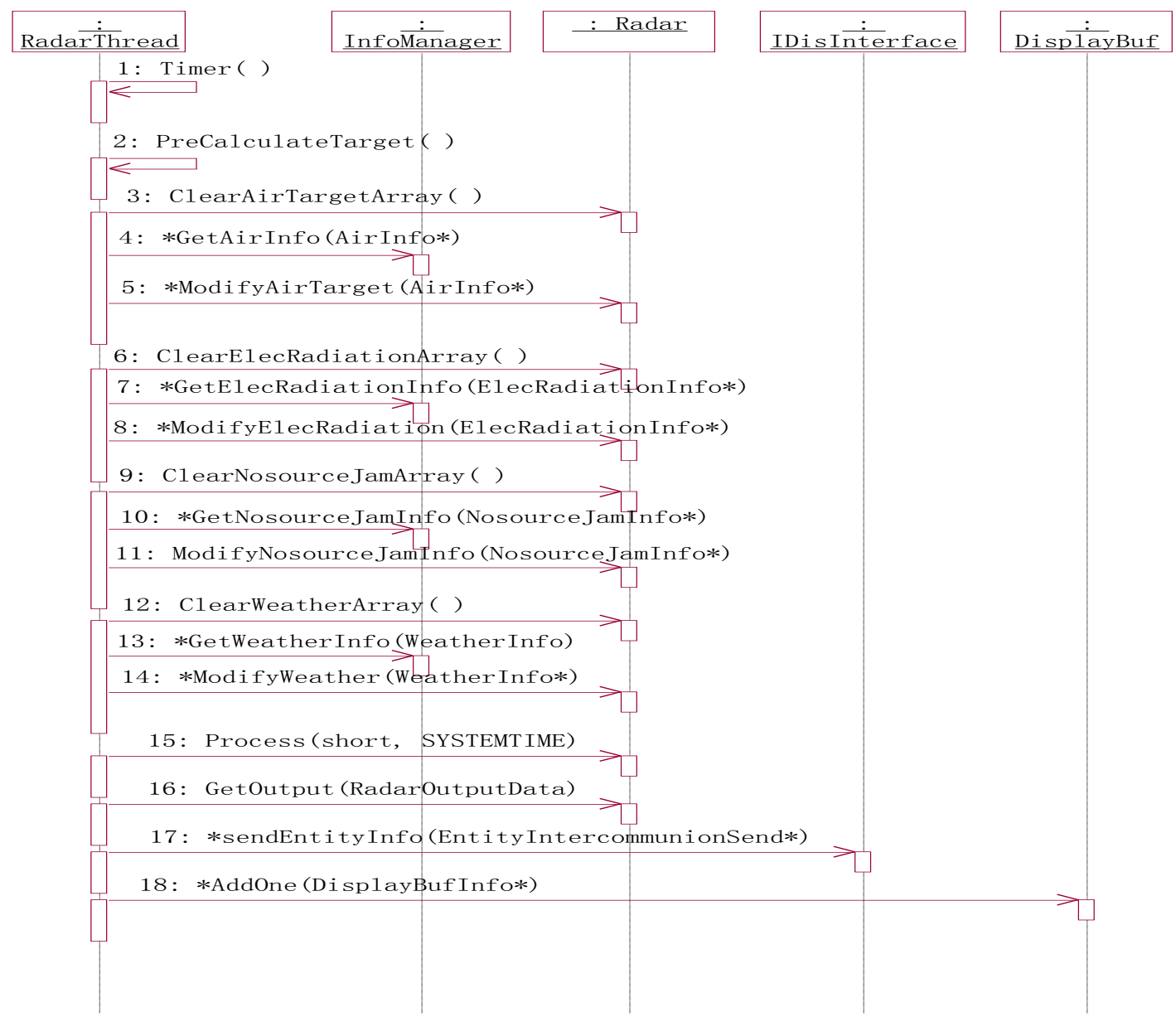

Fig 7 Sequence-diagram of intelligence processing

\section{Conclusion}

In order to simulate and make model for command information system, and realize the reuse of simulated model, it is highly important to standardize the description of model. This article analyses and designs the visualized simulated model of intelligence processing function of command information system by employing UML platform, and it satisfies the communication among commanders, staff, system analysts and software developers, moreover, it designs simulated model in standard process through UML, and eventually makes it possible to reuse the intelligence processing function module in evaluation and research of different requirements.

\section{Reference}

[1] Cao Zhiyao. Designing principle of computer combat simulation system [M]. Bei Jing: The PLA press.1999

[2] Liu Jianxin, Wang Zhongyi. Artillery gunnery [M]. The PLA artillery command college, 1994

[3] Grady Booch,James Rumbaugh,Ivar Jacobson[America], conference:600-603 Weizhong Shao etc. UML user guide [M]. Bei Jing: Mechanical Industry Press, 2001

[4] UML and modeling instruments. Shen Zhou digital training center, 2004.3

[5] Xia Yongchun, Wei Zhang, Yin Guanghong, "Study on synthetical tests of command system", 《System simulation letter》 . 2013.08 